\title{
The Effect of Statin Use on Mortality in Systemic Autoimmune Rheumatic Diseases
}

\author{
April M. Jorge, Na Lu, Sarah F. Keller, Sharan K. Rai, Yuqing Zhang, and Hyon K. Choi
}

ABSTRACT. Objective. Systemic autoimmune rheumatic diseases (SARD) are associated with an increased risk of premature cardiovascular disease (CVD) and all-cause mortality. We examined the potential survival benefit of statin use among patients with SARD in a general population setting.

Methods. We conducted an incident user cohort study using a UK general population database. Our population included patients with a SARD as determined by Read code diagnoses of systemic lupus erythematosus, systemic sclerosis, Sjögren syndrome, dermatomyositis, polymyositis, mixed connective tissue disease, Behçet disease, or antineutrophil cytoplasmic antibodies-associated vasculitis between January 1,2000, and December 31, 2014. We compared propensity score-matched cohorts of statin initiators and noninitiators within 1-year cohort accrual blocks to account for potential confounders, including disease duration, body mass index, lifestyle factors, comorbidities, and medication use.

Results. Of 2305 statin initiators, 298 died during the followup period (mean $5.1 \mathrm{yrs}$ ), whereas among 2305 propensity score-matched noninitiators, 338 died during the followup period (mean 4.8 yrs). This corresponded to mortality rates of $25.4 / 1000$ and 30.3/1000 person-years, respectively. Statin initiation was associated with reduced all-cause mortality (HR 0.84, 95\% CI 0.72-0.98). When we compared the unmatched cohorts, the statin initiators $(\mathrm{n}=2863)$ showed increased mortality (HR $1.85,95 \%$ CI $1.58-2.16)$ compared with noninitiators $(\mathrm{n}=2863$ randomly selected within 1-year cohort accrual blocks) because of confounding by indication.

Conclusion. In this general population-based study, statin initiation was shown to reduce overall mortality in patients with SARD after adjusting for relevant determinates of CVD risk. (First Release September 1 2018; J Rheumatol 2018;45:1689-95; doi:10.3899/jrheum.171389)

Key Indexing Terms:

RHEUMATIC DISEASES

PREVENTIVE MEDICINE

\section{SYSTEMIC LUPUS ERYTHEMATOSUS}

\section{MORTALITY EPIDEMIOLOGY}

Systemic autoimmune rheumatic diseases (SARD) are associated with premature mortality, largely through an increased risk of premature cardiovascular disease $(\mathrm{CVD})^{1,2,3,4,5,6}$. These conditions share the commonalities of chronic systemic inflammation as well as treatment strategies often involving glucocorticoids and other chronic immunosuppressive therapies. Moreover, they are typically managed

From the Division of Rheumatology, Allergy, and Immunology,

Massachusetts General Hospital, Harvard Medical School, Boston, Massachusetts, USA

This project was supported in part by the Ruth L. Kirschstein Institutional National Research Service Award T32-AR-007258 and National Institutes of Health Grant P60-AR-047785.

A.M. Jorge, MD, Graduate Assistant, Research Fellow, Massachusetts General Hospital, and Harvard Medical School; N. Lu, MPH, Research Assistant, Massachusetts General Hospital; S.F. Keller, MD,

Rheumatology Fellow, Research Fellow, Massachusetts General Hospital, and Harvard Medical School; S.K. Rai, MSc, Research Assistant, Massachusetts General Hospital; Y. Zhang, DSc, Faculty, Harvard Medical School; H.K. Choi, MD, PhD, Professor, Harvard Medical School.

Address correspondence to Dr. A.M. Jorge, Division of Rheumatology, Allergy, and Immunology, Department of Medicine, Massachusetts General Hospital, 55 Fruit St., Bulfinch 165, Boston, Massachusetts 02114, USA.E-mail:AMJorge@mgh.harvard.edu

Accepted for publication May 11, 2018. longitudinally by rheumatologists who often take an active role in the management of comorbidities. In systemic lupus erythematosus (SLE), many studies have shown an increased risk of CVD, including a doubled risk of stroke and myocardial infarction (MI) in the Nurse's Health Study and other large studies $7,8,9,10,11,12$, as well as doubled risk of overall mortality ${ }^{13}$. A large UK population-based study in systemic sclerosis $(\mathrm{SSc})$ found 2.6 times the risk of stroke and 1.8 times the risk of MI relative to peers ${ }^{14}$. Similarly, in granulomatosis with polyangiitis, a large population-based study in British Columbia, Canada, found a 1.5-times increased risk of stroke and a 1.8-times increased risk of $\mathrm{MI}^{15}$, and a recent metaanalysis of observational studies found 2.7-fold increased mortality associated with antineutrophil cytoplasmic antibody-associated vasculitis $(\mathrm{AAV})^{16}$. In myositis, overall mortality is increased, and mounting evidence suggests premature CVD risk, as a previous large population-based study found 1.7-times increased risk of stroke and 3.9-times increased risk of MI in dermatomyositis (DM) and polymyositis (PM) ${ }^{17,18,19}$. With substantial evidence for a collectively higher risk of CVD and mortality for patients with SARD, investigations into strategies to mitigate this risk are urgently needed.

Personal non-commercial use only. The Journal of Rheumatology Copyright @ 2018 . All rights reserved 
In the general population, a major strategy for reducing the risk of CVD and mortality is treatment with statins. A wide body of literature supports the efficacy of statins for primary and secondary prevention of CVD and associated mortality in the general population. Briefly, a Cochrane review of statin use for CVD prevention in the general population found a $25 \%$ reduction in CVD events, and a previous metaanalysis of statin use in the general population found a $9-14 \%$ reduction in overall mortality ${ }^{20,21}$. However, given the rarity of these diseases, evidence regarding the potential mortality benefit of statins among patients with individual SARD is scarce. To help address this gap in knowledge, we conducted a proof-of-concept analysis of the potential survival benefit of statins collectively among individuals with SARD from a large general population database.

\section{MATERIALS AND METHODS}

Data source. The setting for our study was The Health Improvement Network (THIN), an electronic medical record database that represents $6.2 \%$ of the UK population, including over 11 million patients. The THIN database is representative of the general UK population in demographics and the prevalence of common medical diagnose ${ }^{22}$. Health information included in this database includes demographics, lifestyle factors, information from general practitioner (GP) visits, diagnoses from hospital admissions and specialists, medications, and laboratory results. The specific diagnoses are recorded by the Read code classification system, which is the standard nomenclature of clinical terms used by the National Health Service in describing clinical diagnoses ${ }^{23}$. Medication prescriptions are recorded by the Multifunctional Standardized Lexicon for European Community Language (Multilex) classification system ${ }^{24}$.

Study design and cohort definition. We conducted a population-based incident user cohort study. The classification of SARD has been previously described $^{2,25}$. We identified subjects with SARD, defined by having at least 1 Read code for one of the included conditions: SLE, SSc, Sjögren syndrome (SS), DM, PM, mixed connective tissue disease, AAV, or Behçet disease ${ }^{2,25}$. We did not include individuals with diagnoses of giant cell arteritis or polymyalgia rheumatica to improve comparability within the SARD cohort, because those conditions exclusively affect an older age group ${ }^{26}$. Read code diagnoses have been previously validated in a similar UK database, and diagnoses of connective tissue diseases were found to be $80 \%$ accurate $^{27}$.

Within this defined population with SARD, we identified subjects who initiated statin medications between January 1, 2000, and December 31, 2014. To account for potential secular trends in statin prescribing in SARD, subjects were divided into 1-year accrual blocks during this time frame. Within each accrual block, statin initiators were defined as patients with new statin prescriptions during that 1 -year period. To be considered a statin initiator, a subject also had to be enrolled in the THIN database for at least 1 year prior to the first recorded statin prescription. The comparators were subjects with SARD matched 1:1 by the 1-year accrual blocks of entry into the cohort and who did not initiate statin medications during the study period using a 5-to-1 digit "greedy matching" algorithm ${ }^{28,29}$. The index date was the date of statin initiation for statin users and a random date within the accrual year for noninitiators ${ }^{30}$. Individuals were excluded if they were current or prior statin users (i.e., not incident users) or if they had incomplete records of covariates.

Because we expected there would be systematic differences in the baseline characteristics of subjects receiving statin therapy compared to those not prescribed statins, we performed propensity score matching to adjust for these potential imbalances. We calculated propensity scores (the predicted probability of statin initiation) within each 1-year accrual block by logistic regression. The variables included in the propensity score estimation comprised demographics (age, sex), body mass index (BMI), alcohol and tobacco use, healthcare use (as measured by the number of GP visits, specialist referrals, and hospitalizations), specific SARD diagnosis, SARD duration prior to the index date, medication use, comorbid conditions (determined by Read code diagnoses), and cholesterol levels assessed within 1 year prior to the index date of entry into the cohort (Table 1).

Assessment of outcome. The primary outcome of interest was all-cause mortality. This was assessed by the death date automatically recorded in the THIN database when death is registered in the Personal Demographics Service database, which contains demographic data for all patients registered with the National Health Service in the UK. This automatic update has been shown to be an accurate reflection of national death rates in the $\mathrm{UK}^{22}$.

Statistical analysis. Descriptive statistics were calculated for baseline characteristics of the statin initiators and noninitiator comparators in both the propensity score-matched and unmatched cohorts. We calculated person-years (PY) of followup for each subject from the index date until either death, end of the study period, or disenrollment from the THIN database. Subjects remained grouped as "statin initiators" regardless of continuation of the statin prescription. This is analogous to an intention-to-treat analysis used in clinical trials and provides conservative estimates for the target effect. Nevertheless, to examine the potential effect of discontinuation of statins among initiators over time, we performed analyses with the followup time truncated at 1,2,3, and 4 years for all subjects. We generated survival plots with estimates of cumulative mortality over time. We then used Cox proportional hazard models to estimate the effect of statin initiation on mortality, stratified by 1 -year cohort accrual blocks. To examine the potential effect of individual SARD on our effect estimation, we repeated our analysis by excluding 1 subset of all SARD at a time.

This study was approved by the Partners Human Research Committee, approval number 2017P000399. Informed consent was waived because all data were anonymous.

\section{RESULTS}

Unmatched analysis. We identified 2863 statin initiators and 2863 noninitiators in the unmatched cohort with complete covariate information (Table 1). An additional 475 statin initiators were excluded because of missing covariates; their baseline covariates did not differ from the included patients (Supplementary Table 1, available with the online version of this article). Statin initiators had higher baseline cholesterol levels and were older, more often male, and had greater comorbidities. There were 232 deaths among statin initiators over a mean followup time of 3.1 years. The overall mortality rate was 26.0 deaths per $1000 \mathrm{PY}$. In the comparator group, there were 123 deaths over a mean of 3.1 years, with an overall mortality rate of 13.8 deaths per 1000 PY. In this unmatched analysis without controlling for confounding by indication, statin initiation was associated with an overall HR for mortality of 1.85 (95\% CI 1.58-2.16) and greater cumulative mortality as depicted in Figure 1.

Propensity score-matched analysis. In the propensity scorematched cohorts (2305 statin initiators and 2305 noninitiators), subjects were well-balanced by age, sex, BMI, SARD diagnosis, disease duration, alcohol and tobacco use, comorbidities, medication use, and cholesterol levels at baseline (Table 1). SLE represented the largest subset of all SARD, followed by SS and SSc. There were 298 deaths

Personal non-commercial use only. The Journal of Rheumatology Copyright $\odot$ (2018. All rights reserved 
Table 1. Baseline characteristics of statin initiators and noninitiators in the propensity score-matched and unmatched cohorts. Values are $\%$ unless otherwise specified.

\begin{tabular}{|c|c|c|c|c|}
\hline \multirow[t]{2}{*}{ Baseline Characteristics } & \multicolumn{2}{|c|}{ Propensity Score-matched } & \multicolumn{2}{|c|}{ Unmatched* } \\
\hline & Statin Initiators, $n=2305$ & Noninitiators, $\mathrm{n}=2305$ & Statin Initiators, $n=2863$ & Noninitiators, $\mathrm{n}=2863$ \\
\hline \multicolumn{5}{|l|}{ Demographics } \\
\hline Sex, $\%$ male & 23.9 & 23.1 & 24.4 & 16.3 \\
\hline BMI, $\mathrm{kg} / \mathrm{m}^{2}$, mean & 27.6 & 27.6 & 27.5 & 26.0 \\
\hline \multicolumn{5}{|l|}{ SARD } \\
\hline Sjögren syndrome & 30.1 & 28.7 & 29.6 & 26.5 \\
\hline $\mathrm{DM} / \mathrm{PM}$ & 8.1 & 7.8 & 7.8 & 7.8 \\
\hline AAV & 4.2 & 4.4 & 4.7 & 3.1 \\
\hline Behçet disease & 3.9 & 3.8 & 3.9 & 5.8 \\
\hline Disease duration, yrs, mean & 12.5 & 12.5 & 12.4 & 10.7 \\
\hline \multicolumn{5}{|l|}{ Smoking status } \\
\hline Current smokers & 17.7 & 18.1 & 19.2 & 21.2 \\
\hline Antihypertensive medications & 70.2 & 71.8 & 72.6 & 32.9 \\
\hline$\beta$ blockers & 22.9 & 23.1 & 25.9 & 10.0 \\
\hline Calcium channel blockers & 32.5 & 32.5 & 32.9 & 13.8 \\
\hline Nitrates & 8.4 & 8.5 & 10.6 & 8.5 \\
\hline ACE inhibitors & 29.7 & 29.8 & 31.5 & 9.8 \\
\hline NSAID & 29.8 & 30.4 & 29.9 & 28.6 \\
\hline Loop diuretics & 13.5 & 14.4 & 14.8 & 6.5 \\
\hline Thiazide & 21.0 & 21.3 & 20.5 & 9.4 \\
\hline Potassium-sparing diuretics & 4.6 & 5.0 & 5.0 & 2.3 \\
\hline Insulin & 2.8 & 2.9 & 2.9 & 0.5 \\
\hline Anticoagulants & 6.6 & 7.0 & 6.9 & 4.2 \\
\hline Glucocorticoids & 27.7 & 27.3 & 29.4 & 23.8 \\
\hline \multicolumn{5}{|l|}{ Comorbid conditions } \\
\hline Angina & 8.4 & 8.2 & 9.6 & 2.0 \\
\hline Other cardiovascular disease & 3.1 & 3.1 & 3.1 & 0.8 \\
\hline Venous thromboembolism & 6.6 & 7.0 & 6.5 & 5.1 \\
\hline Varicose veins & 12.5 & 12.9 & 11.9 & 10.2 \\
\hline Hyperlipidemia & 18.9 & 18.7 & 20.3 & 26.5 \\
\hline Diabetes & 17.7 & 18.8 & 17.5 & 3.3 \\
\hline $\mathrm{CKD}, \geq$ stage 3 & 11.8 & 12.8 & 11.5 & 5.0 \\
\hline Liver disease & 4.4 & 4.8 & 4.4 & 3.8 \\
\hline Cancer & 11.4 & 11.1 & 10.9 & 9.7 \\
\hline COPD & 6.1 & 6.2 & 6.1 & 3.7 \\
\hline Infection/pneumonia & 11.6 & 11.4 & 12.1 & 9.0 \\
\hline Depression & 18.8 & 18.7 & 18.5 & 17.2 \\
\hline \multicolumn{5}{|l|}{ Healthcare use ${ }^{* *}$} \\
\hline General practice visits & 15.6 & 15.8 & 15.4 & 11.4 \\
\hline Specialist referrals & 1.2 & 1.2 & 1.2 & 0.9 \\
\hline Hospitalizations & 0.9 & 0.9 & 0.9 & 0.6 \\
\hline \multicolumn{5}{|l|}{ Laboratory measurements } \\
\hline Total cholesterol, $\mathrm{mg} / \mathrm{dl}$, mean & 233.5 & 233.8 & 235.6 & 205.5 \\
\hline
\end{tabular}

* A noninitiator was randomly selected for each statin initiator within 1-year accrual blocks. ${ }^{\dagger}$ Over $95 \%$ of aspirin users were taking cardiovascular dosages of aspirin. ** Frequency during the past 2 years. BMI: body mass index; SARD: systemic autoimmune rheumatic disease; SLE: systemic lupus erythematosus; SSc: systemic sclerosis; ANCA: antineutrophil cytoplasmic antibodies; ACE: angiotensin-converting enzyme; NSAID: nonsteroidal antiinflammatory drugs; CKD: chronic kidney disease; COPD: chronic obstructive pulmonary disease; DM: dermatomyositis; PM: polymyositis; AAV: ANCA-associated vasculitis. 


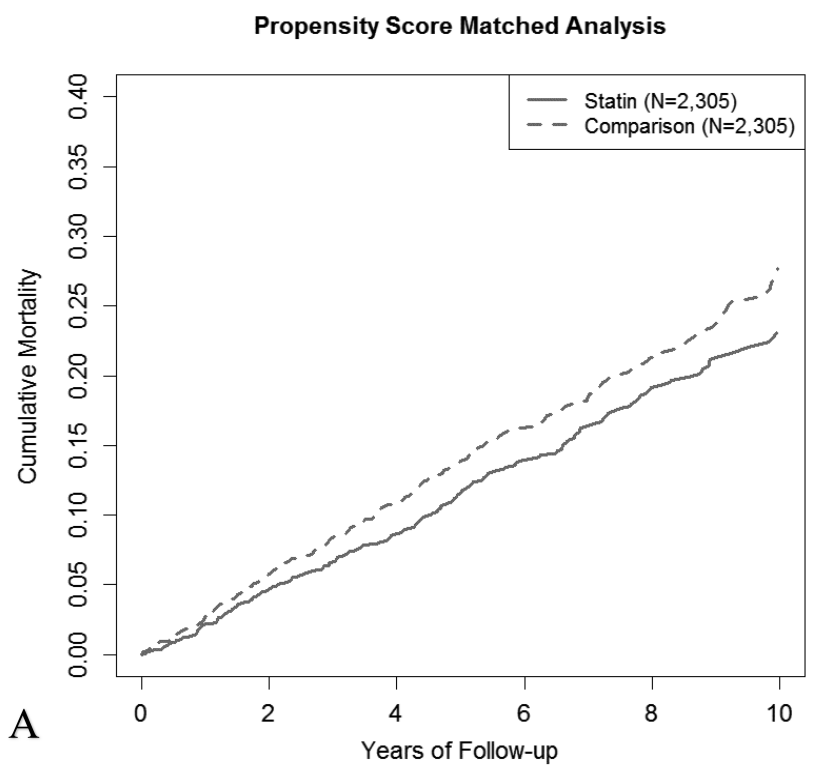

Figure 1. Cumulative mortality over time in statin initiators and noninitiators.

among statin initiators over a mean followup time of 5.1 years. The overall mortality rate was 25.4 deaths per 1000 PY. In the comparator group, there were 338 deaths over a mean of 4.8 years, with an overall mortality rate of 30.3 deaths per $1000 \mathrm{PY}$. Figure 1A depicts the cumulative overall mortality for the 2 exposure groups. The HR for overall mortality associated with statin initiation was 0.84 (95\% CI $0.72-0.98$ ) over the complete followup period. With the truncated followup of $1,2,3$, and 4 years, the overall mortality HR remained $<1$ for statin initiation, trending toward significance for 1 and 2 years of followup, and with HR 0.78 (95\% CI 0.62-0.98) for 3 years of followup and 0.77 (95\% CI 0.63-0.95) for 4 years of followup (Table 2).

Subgroup and sensitivity analysis. In our subgroup analyses, mortality HR associated with statin initiation was $0.81(95 \%$ CI 0.69-0.96) among patients $\geq 55$ years and 0.89 (95\% CI $0.45-1.66)$ among patients $<55$ years. Females, comprising the majority (76\%), had overall mortality HR of 0.77 (95\% CI 0.64-0.92) among statin initiators, whereas among males it was 1.05 (95\% CI 0.77-1.43). The corresponding HR were 0.82 (95\% CI 0.66-1.01) among patients without a prior

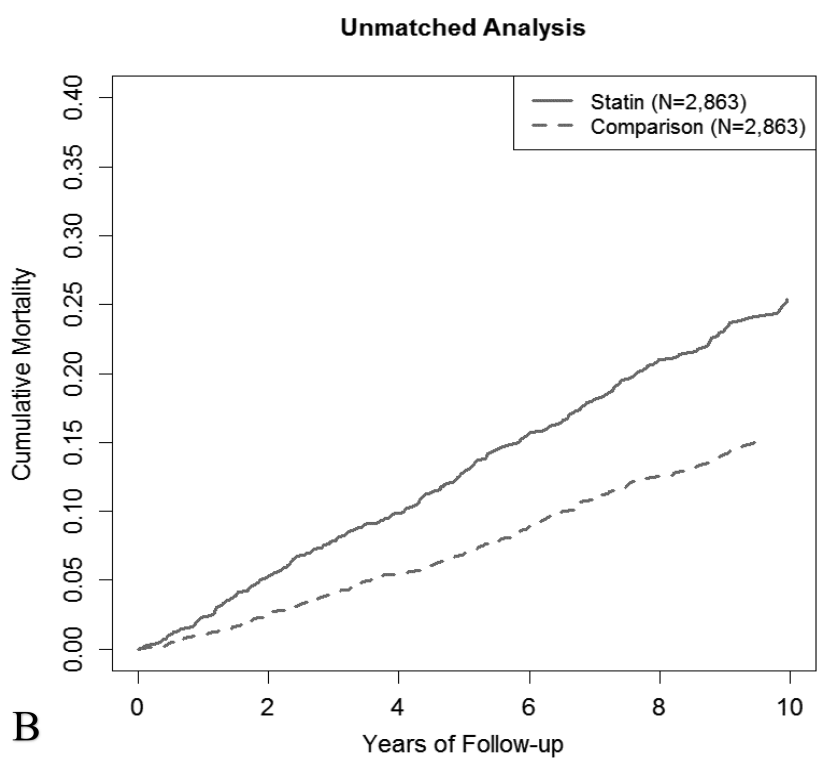

history of CVD (e.g., coronary artery disease, stroke, or peripheral vascular disease) at baseline and 0.81 (95\% CI $0.65-1.02)$ among patients with such conditions. For users of nonsteroidal antiinflammatory drugs and nonusers, the corresponding HR were 0.90 (95\% CI 0.67-1.21) and 0.80 (95\% CI 0.67-0.97), respectively (data not shown).

In our analyses per specific SARD subgroups, the mortality HR were 0.83 (95\% CI 0.66-1.06) for patients with SLE, 0.87 (95\% CI 0.66-1.16) for those with SS, and 0.63 (95\% CI 0.42-0.94) for those with SSc. The numbers of subjects with DM, PM, AAV, and Behçet disease were small (Table 1), limiting meaningful individual subgroup analyses. With the exclusion of 1 subset of all SARD at a time from the propensity score-matched cohorts, the effect estimates of the all-cause mortality reduction associated with statin initiation were similar (HR $0.82,95 \%$ CI $0.70-0.96$ excluding vasculitides; HR $0.82,95 \%$ CI $0.69-0.96$ excluding myositis; HR $0.83,95 \%$ CI $0.68-1.02$ excluding SLE; HR 0.82, $95 \%$ CI $0.68-0.99$ excluding SS, and HR $0.88,95 \%$ CI $0.75-1.05$ excluding SSc; Supplementary Table 2, available with the online version of this article).

Table 2. Association between statin initiation and all-cause mortality in propensity score-matched cohorts.

\begin{tabular}{|c|c|c|c|c|c|}
\hline Followup Period, Yrs & Deaths, $\mathrm{n}$ & Mortality Rate/1000 PY & Deaths, $\mathrm{n}$ & Mortality Rate/1000 PY & HR $(95 \%$ CI) \\
\hline 1 & 46 & 21.1 & 57 & 26.5 & $0.80(0.54-1.18)$ \\
\hline 3 & 129 & 22.6 & 162 & 29.0 & $0.78(0.62-0.98)$ \\
\hline 4 & 160 & 22.4 & 201 & 29.0 & $0.77(0.63-0.95)$ \\
\hline Total followup & 298 & 25.4 & 338 & 30.3 & $0.84(0.72-0.98)$ \\
\hline
\end{tabular}

PY: person-years. 


\section{DISCUSSION}

In this large-scale UK general population-based cohort study of subjects with SARD, statin initiation was associated with a $16 \%$ reduction of all-cause mortality, compared with propensity score-matched patients with SARD who were not treated with statins. This reduction in overall mortality became significant at 3 years of followup and was sustained during the rest of the study followup. The magnitude of the protective association between statin use and mortality risk reduction was similar to that seen in the general population and appeared to be similar across the included individual SARD conditions as well as for primary and secondary CVD prevention indications, although subgroup analysis was limited by sample size 20,21 .

The survival benefit associated with statin initiation in patients with SARD may be related to the pleotropic antiinflammatory effects of statins, in addition to their cholesterol-lowering and antiatherosclerotic effects ${ }^{31}$. Statins have immunomodulatory properties such as altering the function of antigen-presenting cells and $\mathrm{T}$ cells, which may provide a direct benefit in these autoimmune diseases ${ }^{32}$. They have been shown to reduce C-reactive protein levels in patients with $\mathrm{SLE}^{33}$ and may play a role in reducing proteinuria ${ }^{34}$. In SSc, statins have been implicated in reducing endothelial scarring and reducing the development of digital ulcers ${ }^{35}$. One study found simvastatin to reduce neutrophil degranulation in patients with ANCA-associated vasculitis ${ }^{36}$, suggesting a potential role in reducing vascular damage in this disease as well.

Additionally, as newer treatments have improved our ability to control disease activity for patients with SLE, AAV, and other SARD, mortality because of the underlying diseases has declined over time ${ }^{16,37}$. However, these patients continue to experience multiple risk factors for premature atherosclerosis, including side effects from chronic glucocorticoid exposure ${ }^{38,39}$ and an increase in traditional CVD risk factors such as smoking and a sedentary lifestyle ${ }^{40}$, in addition to longstanding chronic inflammation because of the SARD. Therefore, this population at heightened risk of developing CVD may benefit from the effects of statins on reducing atherosclerosis as well as broader antiinflammatory effects $^{41,42}$.

Previous randomized trials of patients with SLE (N ranging from 60 to 221) have investigated the potential effect of statins on surrogate imaging endpoints of atherosclerosis [i.e., carotid plaque, carotid intima media thickness, and coronary artery calcium (CAC)] with conflicting results ${ }^{41}$. While 3 trials did not demonstrate an effect of statin therapy on the subclinical atherosclerosis endpoints ${ }^{43,44,45}, 1$ study showed a reduced progression of CAC in the atorvastatintreated group ${ }^{46}$. These studies had limited sample sizes for clinical endpoints such as mortality. A previous Taiwanese study has assessed mortality risk among SLE patients with hyperlipidemia, but the study did not use an incident user design (as was done in ours) ${ }^{47}$, which is critically important to ensure validity in pharmacoepidemiology ${ }^{48}$. Further, that study's calculation of person-time in relation to SLE onset and duration is highly suggestive of immortal time bias ${ }^{49}$, which would lead to a very protective effect as reported (i.e., mortality HR of high-dose statin use compared with nonusers $=0.45,95 \%$ CI $0.25-0.84)^{47}$. We are not aware of any other studies investigating the mortality effect of statins in patients with SLE or other SARD. Thus our study provides data to suggest the efficacy of statins in reducing mortality for patients with SARD.

Our study has several strengths and limitations that warrant recognition. We used a large general population database, which made our study generalizable and allowed us to identify large numbers of individuals with these rare conditions who have sufficient followup to investigate the outcome of mortality. In considering the individual SARD diagnoses together in 1 analysis, we improved our power to detect overall differences in outcomes. However, our study was not powered to specify the relative effect of statins on mortality in each specific SARD condition. To that end, confirming our findings in individual SARD in a much larger dataset or pooled analyses with multiple datasets would be valuable. In particular, further data on the safety of statins among patients with DM and PM would be useful. Until then, the potential concern of concomitant statin myopathy may outweigh the benefit other than in high cardiovascular riskdyslipidemic patients with these conditions. Nonetheless, our findings demonstrating the benefit of statins in reducing mortality among a collective SARD cohort provides important preliminary evidence. We did not demonstrate the same benefit among males. While this may imply a subgroup effect by sex, the proportion of males was small, limiting meaningful analysis. Further studies would be needed in a larger cohort of males with SARD. Our source population derives from a GP-driven medical record database, and it does not include accurate information regarding disease activity. Therefore, we could not determine the duration of disease flares or details of specific disease manifestations such as Raynaud phenomenon or digital ulcers among patients with SSc. However, it does include numbers of GP visits, hospital admissions, and specialist referrals, which we used to measure healthcare use as a surrogate of illness severity. For ascertainment of the exposure, we relied upon medication prescribing data and could not assess compliance with the statin medications. However, this would tend to provide a conservative estimate of the effect of statin treatment. We considered different potency statins and dosing regimens together in the analysis because of sample size constraints. Future studies could assess the effect of these differences on mortality in patients with SARD. We focused our analysis on all-cause mortality because this outcome has been shown to be highly reliable in our source database, and we had incomplete data on cause-specific mortality ${ }^{22}$.

Personal non-commercial use only. The Journal of Rheumatology Copyright (C) 2018. All rights reserved. 
Regardless, the demonstration of reduced all-cause mortality is itself critically important, as this endpoint reflects the net health outcome of the overall benefits and risks associated with statin use.

We have shown that statin initiation was associated with a reduction in overall mortality for patients with SARD, with a magnitude of effect similar to that previously seen in the general population. The proper use of statins may be beneficial in reducing premature mortality for patients with SARD.

\section{ACKNOWLEDGMENT}

The work was presented at the 2016 American College of Rheumatology Annual Meeting.

\section{ONLINE SUPPLEMENT}

Supplementary material accompanies the online version of this article.

\section{REFERENCES}

1. Prasad M, Hermann J, Gabriel SE, Weyand CM, Mulvagh S, Mankad R, et al. Cardiorheumatology: cardiac involvement in systemic rheumatic disease. Nat Rev Rheumatol 2015;12:168-76.

2. Bernatsky S, Linehan T, Hanly JG. The accuracy of administrative data diagnoses of systemic autoimmune rheumatic diseases. J Rheumatol 2011;38:1612-6.

3. Breunig M, Kleinert S, Lehmann S, Kneitz C, Feuchtenberger M, Tony HP, et al. Simple screening tools predict death and cardiovascular events in patients with rheumatic disease. Scand $\mathbf{J}$ Rheumatol 2018;47:102-9.

4. Bartoloni E, Alunno A, Gerli R. Hypertension as a cardiovascular risk factor in autoimmune rheumatic diseases. Nat Rev Cardiol 2018;15:33-44.

5. Bartoloni E, Shoenfeld Y, Gerli R. Inflammatory and autoimmune mechanisms in the induction of atherosclerotic damage in systemic rheumatic diseases: two faces of the same coin. Arthritis Care Res 2011;63:178-83.

6. Alenghat FJ. The prevalence of atherosclerosis in those with inflammatory connective tissue disease by race, age, and traditional risk factors. Sci Rep 2016;6:20303.

7. Aviña-Zubieta JA, To F, Vostretsova K, De Vera MA, Sayre EC, Esdaile JM. Risk of myocardial infarction and stroke in newly diagnosed systemic lupus erythematosus: a general population-based study. Arthritis Care Res 2017;69:849-56.

8. Schoenfeld S, Kasturi S, Costenbader K. The epidemiology of atherosclerotic cardiovascular disease among patients with SLE: a systematic review. Semin Arthritis Rheum 2013;43:77-95.

9. Urowitz M, Bookman A, Koehler B, Gordon D, Smythe H, Ogryzlo M. The bimodal mortality pattern of systemic lupus erythematosus. Am J Med 1976;60:221-5.

10. Bartels CM, Buhr KA, Goldberg JW, Bell CL, Visekruna M, Nekkanti S, et al. Mortality and cardiovascular burden of systemic lupus erythematosus in a US population-based cohort. J Rheumatol 2014;41:680-7.

11. Hak AE, Karlson EW, Feskanich D, Stampfer MJ, Costenbader KH. Systemic lupus erythematosus and the risk of cardiovascular disease: results from the nurses' health study. Arthritis Rheumatol 2009;61:1396-402.

12. Lee Y, Choi S, Ji J, Song G. Overall and cause-specific mortality in systemic lupus erythematosus: an updated meta-analysis. Lupus 2016;25:727-34

13. Jorge AM, Lu N, Rai SK, Zhang Y, Choi H. Unchanging premature mortality trends in systemic lupus erythematosus: a general population-based study (1999-2014). Rheumatology 2018; 57:337-44

14. Man A, Zhu Y, Zhang Y, Dubreuil M, Rho YH, Peloquin C, et al. The risk of cardiovascular disease in systemic sclerosis: a population-based cohort study. Ann Rheum Dis 2013;72:1188-93.

15. Aviña-Zubieta JA, Mai A, Amiri N, Dehghan N, Ann Tan J, Sayre EC, et al. Risk of myocardial infarction and stroke in patients with granulomatosis with polyangiitis (Wegener's): a population-based study. Arthritis Rheumatol 2016;68:2752-9.

16. Tan JA, Dehghan N, Chen W, Xie H, Esdaile JM, Avina-Zubieta JA. Mortality in ANCA-associated vasculitis: a meta-analysis of observational studies. Ann Rheum Dis 2017;76:1566-74.

17. Rai SK, Choi HK, Sayre EC, Aviña-Zubieta JA. Risk of myocardial infarction and ischaemic stroke in adults with polymyositis and dermatomyositis: a general population-based study. Rheumatology 2016;55:461-9.

18. Linos E, Fiorentino D, Lingala B, Krishnan E, Chung L. Atherosclerotic cardiovascular disease and dermatomyositis: an analysis of the Nationwide Inpatient Sample survey. Arthritis Res Ther 2013;15:R7.

19. Dobloug GC, Svensson J, Lundberg IE, Holmqvist M. Mortality in idiopathic inflammatory myopathy: results from a Swedish nationwide population-based cohort study. Ann Rheum Dis 2018;77:40-7.

20. Taylor F, Huffman MD, Macedo AF, Moore TH, Burke M, Davey Smith G, et al. Statins for the primary prevention of cardiovascular disease. Cochrane Database Syst Rev 2013:CD004816.

21. Cholesterol Treatment Trialists' (CTT) Collaborators; Mihaylova B, Emberson J, Blackwell L, Keech A, Simes J, Barnes EH, et al. The effects of lowering LDL cholesterol with statin therapy in people at low risk of vascular disease: meta-analysis of individual data from 27 randomised trials. Lancet 2012;380:581-90.

22. Blak BT, Thompson M, Dattani H, Bourke A. Generalisability of The Health Improvement Network (THIN) database: demographics, chronic disease prevalence and mortality rates. Inform Prim Care 2011;19:251-5

23. NHS Digital. Read Codes. [Internet. Accessed June 11, 2018.] Available from: digital.nhs.uk/article/1104/Read-Codes

24. FDB. Multilex. [Internet. Accessed June 11, 2018.] Available from: www.fdbhealth.co.uk/solutions/multilex

25. Armstrong SM, Wither JE, Borowoy AM, Landolt-Marticorena C, Davis AM, Johnson SR. Development, sensibility, and validity of a systemic autoimmune rheumatic disease case ascertainment tool. J Rheumatol 2017;44:18-23.

26. Gonzalez-Gay MA, Pina T. Giant cell arteritis and polymyalgia rheumatica: an update. Curr Rheumatol Rep 2015;17:6

27. Herrett E, Thomas SL, Schoonen WM, Smeeth L, Hall AJ. Validation and validity of diagnoses in the General Practice Research Database: a systematic review. Br J Clin Pharmacol 2010;69:4-14.

28. D'Agostino RB, Rubin DB. Estimating and using propensity scores with partially missing data. J Am Stat Assoc 2000;95:749-59.

29. Austin PC. A comparison of 12 algorithms for matching on the propensity score. Stat Med 2014;33:1057-69.

30. Seeger JD, Walker AM, Williams PL, Saperia GM, Sacks FM. A propensity score-matched cohort study of the effect of statins, mainly fluvastatin, on the occurrence of acute myocardial infarction. Am J Cardiol 2003;92:1447-51.

31. Mihos CG, Artola RT, Santana O. The pleiotropic effects of the hydroxy-methyl-glutaryl-CoA reductase inhibitors in rheumatologic disorders: a comprehensive review. Rheumatol Int 2012;32:287-94.

32. Greenwood J, Steinman L, Zamvil SS. Statin therapy and autoimmune disease: from protein prenylation to immunomodulation. Nat Rev Immunol 2006;6:358-70.

33. Sahebkar A, Rathouska J, Derosa G, Maffioli P, Nachtigal P. Statin

Personal non-commercial use only. The Journal of Rheumatology Copyright @ $\odot$ 2018. All rights reserved 
impact on disease activity and C-reactive protein concentrations in systemic lupus erythematosus patients: a systematic review and meta-analysis of controlled trials. Autoimmun Rev 2016;15:344-53.

34. Ridker PM, MacFadyen J, Libby P, Glynn RJ. Relation of baseline high-sensitivity C-reactive protein level to cardiovascular outcomes with rosuvastatin in the Justification for Use of statins in Prevention: an Intervention Trial Evaluating Rosuvastatin (JUPITER). Am J Cardiol 2010;106:204-9.

35. Kuwana M. Potential benefit of statins for vascular disease in systemic sclerosis. Curr Opin Rheumatol 2006;18:594-600.

36. Al-Ani B. Simvastatin inhibits neutrophil degranulation induced by anti-neutrophil cytoplasm auto-antibodies and N-formylmethionine-leucine-phenylalanine (fMLP) peptide. Saudi Med J 2013;34:477-83.

37. Bernatsky S, Boivin JF, Joseph L, Manzi S, Ginzler E, Gladman DD, et al. Mortality in systemic lupus erythematosus. Arthritis Rheumatol 2006;54:2550-7.

38. Keller SF, Miloslavsky EM. Corticosteroids in antineutrophil cytoplasmic antibody-associated vasculitis. Rheum Dis Clin North Am 2016;42:91-101.

39. Apostolopoulos D, Morand EF. It hasn't gone away: the problem of glucocorticoid use in lupus remains. Rheumatology 2017; 56:i114-22.

40. Pinto AJ, Roschel H, de Sá Pinto AL, Lima FR, Pereira RMR, Silva $\mathrm{CA}$, et al. Physical inactivity and sedentary behavior: Overlooked risk factors in autoimmune rheumatic diseases? Autoimmun Rev 2017;16:667-74.

41. Artola RT, Mihos CG, Santana O. Effects of statin therapy in patients with systemic lupus erythematosus. South Med J 2016;109:705-11.
42. Andrades C, Fuego C, Manrique-Arija S, Fernandez-Nebro A Management of cardiovascular risk in systemic lupus erythematosus: a systematic review. Lupus 2017; 26:1407-19.

43. Petri MA, Kiani AN, Post W, Christopher-Stine L, Magder LS. Lupus Atherosclerosis Prevention Study (LAPS). Ann Rheum Dis 2011;70:760-5.

44. Mok CC, Wong CK, To CH, Lai JP, Lam CS. Effects of rosuvastatin on vascular biomarkers and carotid atherosclerosis in lupus: a randomized, double-blind, placebo-controlled trial. Arthritis Care Res 2011;63:875-83.

45. Schanberg LE, Sandborg C, Barnhart HX, Ardoin SP, Yow E, Evans GW, et al; Atherosclerosis Prevention in Pediatric Lupus Erythematosus Investigators. Use of atorvastatin in systemic lupus erythematosus in children and adolescents. Arthritis Rheumatol 2012;64:285-96.

46. Plazak W, Gryga K, Dziedzic H, Tomkiewicz-Pajak L, Konieczynska M, Podolec P, et al. Influence of atorvastatin on coronary calcifications and myocardial perfusion defects in systemic lupus erythematosus patients: a prospective, randomized, double-masked, placebo-controlled study. Arthritis Res Ther 2011;13:R117.

47. Yu HH, Chen PC, Yang YH, Wang LC, Lee JH, Lin YT, et al. Statin reduces mortality and morbidity in systemic lupus erythematosus patients with hyperlipidemia: A nationwide population-based cohort study. Atherosclerosis 2015;243:11-8.

48. Ray WA. Evaluating medication effects outside of clinical trials: new-user designs. Am J Epidemiol 2003;158:915-20.

49. Levesque LE, Hanley JA, Kezouh A, Suissa S. Problem of immortal time bias in cohort studies: example using statins for preventing progression of diabetes. BMJ 2010;340:b5087. 